\title{
REMARK ON MEASURABLE GRAPH THEOREMS
}

\author{
JÜRGEN LEHN
}

ABSTRACr. A theorem generalizing some known measurable graph theorems is proved.

1. Notations. Two measurable spaces $\left(X_{1}, Q_{1}\right)$ and $\left(X_{2}, Q_{2}\right)$ are isomorphic if there exists a bimeasurable one-to-one function from $X_{1}$ onto $X_{2}$. A measurable space is called a standard measurable space if it is isomorphic with ( $I$, $\mathscr{B}(I))$, the unit interval and its Borel $\sigma$-field. We call it a Blackwell space if it is isomorphic with some space $(A, \mathscr{B}(A))$, where $A$ is an analytic subset of $I$ and $\mathscr{B}(A)$ is the system of all intersections of $A$ with sets in $\mathscr{B}(I)$. We claim that $(X, \mathscr{B}(X))$ is a standard measurable space if $X$ is any nondenumerable complete separable metric space and $\mathscr{B}(X)$ its Borel $\sigma$-field. If $(X, \mathcal{Q}, \mu)$ is a measure space, then we write throughout the paper $Q_{\mu}$ for the completion of $Q$ with respect to $\mu$.

2. Main results. Let $(X, \mathcal{Q})$ and $(Y, \mathscr{B})$ be measurable spaces, $f$ a function $f: X \rightarrow Y, \operatorname{gr}(f)$ the graph of $f$ and $\mathbb{Q} \otimes \mathscr{B}$ the product $\sigma$-field. It is well known that $(\mathscr{Q}, \mathscr{B})$-measurability of the function implies $\operatorname{gr}(f) \in \mathbb{Q} \otimes \mathscr{B}$ if $\Re$ is countably separated (see e.g. [5, I.3.4 and 5]). Concerning the reverse implication we shall prove in this paper two notes:

(A) Let $(X, \mathcal{Q})$ and $(Y, \mathscr{B})$ be Blackwell spaces and $\mu$ be a measure on $\mathbb{Q}$. If $\operatorname{gr}(f) \in \mathbb{Q}_{\mu} \otimes \Re$, then $f$ is $\left(\mathbb{Q}_{\mu}, \mathscr{B}\right)$-measurable.

(B) Let $(X, Q, \mu)$ be a $\sigma$-finite measure space and $(Y, \Re)$ a Blackwell space. If $\operatorname{gr}(f) \in \mathbb{Q}_{\mu} \otimes \mathfrak{B}$, then $f$ is $\left(\mathbb{Q}_{\mu}, \mathfrak{B}\right)$-measurable.

In [3] Buckley has proved a result corresponding to (A) on the more restrictive assumption that the spaces $(X, Q)$ and $(Y, \Re)$ are complete separable metric spaces with their Borel $\sigma$-fields. In the most important case of a $\sigma$-finite measure, Buckley's result is known from Bierlein's measurable graph theorem in [2], where $(Y, \Re)$ is assumed to be a standard measurable space and $(X, \mathbb{Q}, \mu)$ is any $\sigma$-finite measure space. In (B) Bierlein's assumption on $(Y, \mathscr{B})$ is generalized to the assumption of a Blackwell space.

Now (A) and (B) may be applied to give a more general version of Buckley's theorem, of the main part of Bierlein's measurable graph theorem and of the known fact that $\operatorname{gr}(f) \in \mathbb{Q} \otimes \mathscr{B}$ is equivalent to $(\mathbb{Q}, \mathscr{B})$-measura-

Received by the editors May 27, 1976.

AMS (MOS) subject classifications (1970). Primary 28A20.

Key words and phrases. Measurable functions, measurable graph theorems, analytic sets, Blackwell spaces. 
bility of $f$ if both spaces $(X, \mathcal{Q})$ and $(Y, \mathscr{B})$ are Blackwell spaces (cf. [5, II.4.3]). Since $\mathscr{B}$ is countably separated if $(Y, \mathscr{B})$ is a Blackwell space, the following theorem is a consequence of $(\mathrm{A})$ and $(\mathrm{B})$.

Measurable Graph Theorem. Let $(X, \mathcal{Q}, \mu)$ be a measure space, $(Y, \mathscr{B})$ a Blackwell space and $f$ a function $f: X \rightarrow Y$. If either the measure $\mu$ is $\sigma$-finite or the space $(X, \mathcal{Q})$ is a Blackwell space, then the equivalence holds:

$f$ is $\left(\mathbb{Q}_{\mu}, \Re\right)$-measurable if and only if $\operatorname{gr}(f) \in \mathbb{Q}_{\mu} \otimes \Re$.

3. Proofs. The proof of (A) is based on the following lemma. The proof of the lemma is omitted as a standard one.

Lemma. Let $(X, Q, \mu)$ be a measure space and $(Y, \mathscr{B})$ a measurable space. If $G \in \mathbb{Q}_{\mu} \otimes \Re$, then there exists a set $N_{0} \in \mathbb{Q}$ such that $\mu\left(N_{0}\right)=0$ and $G \backslash\left(N_{0} \times Y\right) \in \mathbb{Q} \otimes \Re$.

Proof of (A). Let $(X, \mathbb{Q})$ and $(Y, \Re)$ be Blackwell spaces and $\operatorname{gr}(f) \in \mathbb{Q}_{\mu}$ $\otimes \mathscr{B}$. Then, by the Lemma, $X$ is the union of two disjoint sets $X_{0} \in \mathbb{Q}$ and $N_{0} \in \mathbb{Q}$ such that

$$
\mu\left(N_{0}\right)=0 \text { and } \operatorname{gr}(f) \backslash\left(N_{0} \times Y\right) \in \mathbb{Q} \otimes \mathscr{B} .
$$

Let $Q \cap X_{0}$ be the $\sigma$-field of all intersections of $X_{0}$ with sets of $Q$. Since, by definition, $\left(X_{0}, \mathbb{Q} \cap X_{0}\right)$ is a Blackwell space, too, we can apply the measurable graph theorem for Blackwell spaces (see [5, II.4.3]) to the function $f_{0}$ : $X_{0} \rightarrow Y$ defined as the restriction of $f$ on $X_{0}$. Since $\operatorname{gr}\left(f_{0}\right)=\operatorname{gr}(f) \backslash\left(N_{0} \times Y\right)$ is an element of $\left(Q \cap X_{0}\right) \otimes \mathscr{B}$, by this theorem, $f_{0}$ is an $\left(\Theta \cap X_{0}, \mathscr{B}\right)$ measurable function. Then we have for any $B \in \mathscr{B}$,

$$
\begin{aligned}
f^{-1}(B) & =\left(f^{-1}(B) \cap X_{0}\right) \cup\left(f^{-1}(B) \cap N_{0}\right) \\
& =f_{0}^{-1}(B) \cup\left(f^{-1}(B) \cap N_{0}\right) \in \mathbb{Q}_{\mu},
\end{aligned}
$$

because $f_{0}^{-1}(B) \in \mathbb{Q}, f^{-1}(B) \cap N_{0}$ is a subset of $N_{0}$ and $\mu\left(N_{0}\right)=0$. This shows that $f$ is $\left(\mathbb{Q}_{\mu}, \Re\right)$-measurable, and (A) is proved.

To prove (B) it is possible to apply a projection theorem (II.2.2 in [5] is a suitable one) and Choquet's theorem on capacities (see [4, Theorem 1]) as done by Bierlein [2] in the case of a standard measurable space $(Y, \mathscr{B})$. However, to give a short proof, we claim that (B) is an immediate consequence of the following generalization of Aumann's selection theorem (see [1, p. 17]) proved in [6] (cf. Theorem 3, and for the difference in formulation see [5, III.2.3]):

SElection TheOREM. Let $(X, \mathbb{Q})$ be a measurable space having the property that $Q$ equals its universal completion, $(Y, \mathscr{B})$ be a Blackwell space and $C \in \mathbb{Q} \otimes$. If the projection of $C$ on $X$ is all of $X$, then there exists an $(\mathbb{Q}$, $\Re)$-measurable function $s: X \rightarrow Y$ such that $(x, s(x)) \in C$ for all $x \in X$.

REMARKS. 1. That our theorem generalizes Buckley's theorem [3] and the main part of Bierlein's measurable graph theorem [2] is evident. But our 
theorem generalizes also the known result that for Blackwell spaces $(X, \mathbb{Q})$ and $(Y, \mathscr{B}), \operatorname{gr}(f) \in Q \otimes \mathscr{B}$ is equivalent to $(Q, \mathscr{B})$-measurability of the function $f$. This can be seen if we define the measure $\mu$ on $\mathbb{Q}$ by $\mu=0$ for the void set and $\mu=\infty$ for all other sets in $\mathbb{Q}$.

2. That neither in (A) nor in (B) the assumption on $(Y, \mathscr{B})$ to be a Blackwell space is superfluous can be seen by the following simple example: $X=Y=I$ (the unit interval), $Q$ the Borel sets in $I, \mathscr{B}$ the $\sigma$-field generated by the Borel sets and one set $V$ being nonmeasurable with respect to Lebesgue measure, and $\mu$ the Lebesgue measure on $Q$. If $f$ is the identity on $I$, then $\operatorname{gr}(f) \in \mathbb{Q}_{\mu} \otimes \Re$, but the function is not $\left(\mathbb{Q}_{\mu}, \mathscr{B}\right)$-measurable, since $f^{-1}(V) \notin \mathbb{Q}_{\mu}$.

ACKNOWLEDGments. The author thanks the referee, Professor Tibor Neubrunn, for suggestions that led to an improvement of the paper.

\section{REFERENCES}

1. R. J. Aumann, Measurable utility and measurable choice theorem (Colloq. Internat., 1967), Centre Nat. Recherche Sci., Paris, 1969, pp. 15-26. MR 41 \#6548.

2. D. Bierlein, Der Graph messbarer Funktionen mit abstraktem Definitionsbereich, Math. Z. 76 (1961), 468-471. MR 23 \# A3817.

3. J. J. Buckley, Graphs of measurable functions, Proc. Amer. Math. Śoc. 44 (1974), 78-80. MR 49 \#508.

4. G. Choquet, Forme abstraite du théorème de capacitabilité, Ann. Inst. Fourier Grenoble 9 (1959), 83-89. MR 22 \#3692b.

5. J. Hoffman-Jorgensen, The theory of analytic sets, Various Publication Series 10, Aarhus, 1970.

6. M.-F. Sainte-Beuve, On the extension of von Neumann-Aumann's theorem, J. Functional Analysis 17 (1974), 112-129. MR 51 \# 10564.

Mathematisches Institut, Universität Karlsruhe, D-7500 KarlsRuhe, Federal RepubLIC OF GERMANY 